\title{
Meta
}

Journal des traducteurs

Translators' Journal

\section{NAVARRo Domínguez, Fernando (ed.) (2015): Azorín y Miró en traducción. Alicante: Publicaciones de la Universidad de Alicante, $474 \mathrm{p}$.}

\section{Montserrat Cunillera Domènech}

Volume 62, numéro 2, août 2017

URI : https://id.erudit.org/iderudit/1041040ar

DOI : https://doi.org/10.7202/1041040ar

Aller au sommaire du numéro

Éditeur(s)

Les Presses de l’Université de Montréal

ISSN

0026-0452 (imprimé)

1492-1421 (numérique)

Découvrir la revue

Citer ce compte rendu

Cunillera Domènech, M. (2017). Compte rendu de [NAVARRo DOMíNGUEZ, Fernando (ed.) (2015): Azorín y Miró en traducción. Alicante: Publicaciones de la Universidad de Alicante, 474 p.] Meta, 62(2), 478-481.

https://doi.org/10.7202/1041040ar d'utilisation que vous pouvez consulter en ligne.

https://apropos.erudit.org/fr/usagers/politique-dutilisation/ 
interest, even when he speaks about Hugo, lies in the research of poetry. Taking a closer look, we may conclude that all four articles which constitute the third part of the book are at least two-dimensional. As a scholar whose passion lies in literal studies and precisely in the research of Baudelaire's poetry, Brzozowski cannot help admiring "le génie" of the French Romanticism. On the other hand, as the book is dedicated to translation, the author finds a perfect combination of two different approaches to translation: he pairs linguistic and poetic analysis of the material with the analysis of its translations and their respective place in target cultures and literal systems; in particular - in Polish literature. Thus, the first two articles, dedicated majorly to Baudelaire and to a lesser extent to Verlaine (pp. 213-124), show the author's concern about the poetic analysis of stylistic figures and tropes describing either the urban landscape or the semantic field of "incredible ascension." Conversely, the third article (pp. 235-248) focuses on the polysystemic analysis of the recognition of Hugo's works in Poland. It is astonishing that despite the fact that the novels of Hugo were highly praised by the Polish readership, his poetry was significantly discredited due to certain ideological factors resulting in the cultural alienation of French Romanticism. The last article of the "poetic" partition (pp. 249-262) represents a vivid example of historiographical translatology as it is dedicated to the history of translation and retranslation of Baudelaire's poetry in Poland since its first introduction into Polish literal system in 1876. As a case study, Brzozowski has chosen a sonnet, "À une passante," which had been translated into the Polish language nine times over the period of a century. As a result, a scrupulous poetic and rhythmic analysis of all the nine translations has allowed Brzozowski to draw an important conclusion about poetic translation in Poland: it has been a common rule for Polish translators to modernize the source text by translating the "ancient" poets in regular verses. Undoubtedly, this contributes to our better understanding of Polish tradition of translation.

In the conclusion, "Autour de la traduction" by Brzozowski would be a good choice for complementary reading for anyone interested in translation and particularly in literal translation and, as the author puts it, in its descriptive poetics. Moreover, the book sets up the pathways for research into the ways to combine the multidimensional Western (or rather Anglo-Saxon) Translation studies with Eastern European translatology which seems to maintain some relationships with linguistic structuralism.

Gleb Dmitrienko

Université de Montréal, Montréal, Canada

\section{REFERENCES}

Berman, Antoine. (1995). Pour une critique des traductions: John Donne. Paris: Gallimard.

Brzozowski, Jerzy. (2008). Le problème des stratégies du traduire. Meta: journal des traducteurs, 53(4):765-781.

Chesterman, Andrew. (2005). Problems with strategies. In: Krisztina KÁrolY and Ágota FóRIS, eds. New Trends in Translation Studies. Budapest: Akademiai Kiadó.

Chesterman, Andrew. (2006). Questions in the sociology of translation. In: João Ferreira Duarte, Alexandra Assis Rosa and Teresa SERUYA, eds. Translation Studies at the Interface of Disciplines. Amsterdam/Philadelphia: John Benjamins. 9-27.

Dmitrienko, Gleb. (2015). Vers une science de la traduction? Contextes idéologiques, politiques et institutionnels du développement de la Théorie Linguistique de la Traduction en Russie soviétique (1922-1991). Mémoire de maîtrise en traduction. Université de Montréal, Québec, Canada.

Fedorov, Andrei (1953/2002). Vvedenie v teoriyu perevoda [Introduction to the theory of translation]. Ed. 5. St.Petersbourg/Moscow: Philological department of SPbGU / Philologia Tri.

Komissarov, Vilen. (2002). Lingvisticheskoe perevodovedenie $v$ Rossii [Linguistic translation in Russia]. Moscow: ETS.

Mauranen, Anna and Kujamäki, Pekka, eds. (2004). Translation Universals. Do they exist? Amsterdam: John Benjamins.

Monti, Enrico and Schnyder, Peter, eds. (2012). La retraduction. Perspectives littéraires européennes. Avec un texte inédit de Jean-René Ladmiral. Paris: Orizons.

RetSKer, Yakov. (1974). Teoria perevoda i perevodcheskaja praktika [The theory and practice of translation]. Moscow: Mezhdunarodnye otnosheniya.

Shvejcer, Alexander. (1973). Perevod i lingvistika: Uchebnoe posobie dlya perevodchikov [Translation and Linguistics: Manual for translators]. Moscow: Voenizdat.

Simeoni, Daniel. (2007). Translation and society: The emergence of a conceptual relationship. In: Paul St-Pierre and Prafulla C. KAR, eds. Translation - Reflections, Refractions, Transformations. Amsterdam/Philadelphia: John Benjamins, 13-26

Navarro Domínguez, Fernando (ed.) (2015): Azorín y Miró en traducción. Alicante: Publicaciones de la Universidad de Alicante, 474 p.

Azorín y Miró en traducción es una obra colectiva, dirigida y coordinada por el profesor Fernando Navarro y editada por la Universidad de Alicante. Tal y como se indica en el mismo prólogo, recoge 
un amplio abanico de estudios de crítica literaria y traducción a diferentes lenguas de las obras de los escritores alicantinos de la primera mitad del siglo XX: Azorín y Gabriel Miró.

El libro está dividido en tres partes. La primera parte, formada por cuatro artículos, está dedicada a las figuras de Azorín y Miró en la literatura española y al repertorio comentado de todas sus traducciones a diversas lenguas europeas. En el primer artículo, Miguel Ángel Lozano, catedrático de Literatura y autor de las obras completas de Azorín y Miró en Espasa-Calpe y en la Fundación Castro de Madrid, reflexiona de manera magistral sobre la singularidad de ambos escritores y sobre sus aportaciones en el desarrollo de la historia literaria contemporánea, situándolos como figuras destacadas del Modernismo. En el segundo artículo, Fernando Navarro, coordinador del volumen y catedrático de Traducción e Interpretación, lleva a cabo un riguroso trabajo de recopilación de las traducciones de las obras de estos escritores a varias lenguas europeas (alemán, danés, checo, francés, inglés, italiano, lituano, neerlandés, noruego, rumano, ruso, serbio, sueco) y da a conocer el nivel de conocimiento de dichos escritores en las literaturas europea y norteamericana. En el tercer capítulo, José Payá e Iván Martínez presentan un balance de las distintas traducciones de la obra de Azorín que componen el fondo archivístico de la Casa-Museo Azorín así como de otras versiones de las que se tiene constancia. Y en el cuarto estudio, Yolanda Sánchez, directora de la Biblioteca de G. Miró de Alicante, examina la relevancia archivística de esta biblioteca y su doble rol como biblioteca pública y biblioteca de investigación consagrada a la memoria de Gabriel Miró. Asimismo, ofrece una descripción detallada de las novelas traducidas de Miró que constan en la biblioteca y un esbozo de su tarea como traductor, mostrando el interés de los mencionados catálogos como herramienta de investigación para los Estudios de Traducción.

La segunda parte es la más extensa; está formada por veinte artículos en los que se analizan, partiendo de metodologías o marcos teóricos diferentes, traducciones de doce obras de Azorín y ocho obras de Miró al alemán, francés, inglés, checo, neerlandés, rumano y ruso. Diez investigadores poseen como lengua materna la lengua a la que se ha traducido la obra de Azorín o Miró: W. Pöckl, A. Allaigre, P. Masseau, D. Bell, J. Krolová, V. Obdržálkova, H. Gevers, D. Prodan, A. Mioara y N. Timosenko. Y otros diez tienen como lengua materna el español; son profesores e investigadores en diferentes universidades españolas en las lenguas y culturas objeto de estudio en el trabajo traductológico aquí reseñado: J. A. Albadalejo, P. Valero, A. Gómez, J. J. Zaro, M. Tolosa, P. Blanco, J. Jover, A.B. González, A. Olivares y F. Torres. Esta publicación tiene pues el gran mérito de reunir una gran diversidad de especialistas, combinaciones lingüísticas y enfoques metodológicos en el estudio de la obra de los dos escritores españoles mencionados.

Tres son los estudios que se centran en la traducción al alemán de Azorín. En el primero, Wolfgang Pöckl, presenta un análisis minucioso de tres tipos de dificultades con que se enfrentó la traductora, como son la sufijación apreciativa, las referencias culturales del ámbito religioso y las citas literarias en latín y español. En el siguiente, Juan Antonio Albaladejo analiza en qué medida las estrategias traslativas empleadas por los traductores consiguen recrear los rasgos estilísticos peculiares del escritor español y qué elementos se han perdido a causa de los anisomorfismos lingüístico-culturales. Por su parte, Pino Valero, basándose en el cuento Los niños en la playa ('Kinder am Strand')", profundiza en la traducción de un género poco cultivado por el escritor español, pero que resulta clave para comprender su producción literaria.

Siguen ocho estudios dedicados a la traducción al francés de las obras de Azorín y Miró. Annick Allaigre compara dos lecturas en francés de la obra El obispo leproso de Miró: una traducción que presenta una visión psicológica de las situaciones y una traducción que tiende a objetivarlas sistemáticamente. A continuación, Pilar Blanco atribuye el resultado excelente de la traducción francesa de La ruta de Don Quijote de Azorín (Les traces de Don Quichotte) al buen conocimiento que el traductor Christian Manso posee de todo lo relacionado con el mundo quijotesco y su entorno, si bien echa en falta el recurso a equivalentes funcionales. Ana Belén González contrasta el estilo descriptivo y detallista de la novela Doña Inés de Azorín con el estilo de la traducción francesa de Georges Pillement, demasiado literal, con expresiones poco naturales en lengua meta, que alteran el grado de formalidad del registro original o comportan contrasentidos, falsos sentidos y supresiones. En el siguiente estudio, Yolanda Jover compara con minuciosidad la novela Félix Vargas. Etopeya de Azorín con la traducción realizada por Francis de Miomandre, poniendo de manifiesto el trabajo excelente de reescritura que el poeta francés llevó a cabo. Por su parte, Paola Masseau, en un interesante estudio contrastivo, muestra que la traductora francesa consigue recrear las peculiaridades estilísticas de la La ruta de Don Quijote mediante una extrema literalidad. Amparo Olivares destaca la enorme dificultad que supone traducir el trabajo estilístico de Las cerezas del cementerio de Miró y constata la inevitable pérdida de colorido local y expresividad en la versión francesa Les cerises du cimetière. Miguel Tolosa 
se aproxima al proceso traductor que presidió el trabajo del escritor y traductor francés Valéry Larbaud al traducir El humo dormido de Miró, basándose en información extraída de la relación epistolar que ambos mantuvieron y que le permite reflexionar sobre la extrema complejidad que supone traducir la obra de Miró a causa de su óptica emotiva. Francisco Torres se interesa por los problemas estructurales y estilísticos que plantea la traducción del texto Castilla de Azorín, y mediante un análisis comparativo muestra el empobrecimiento de la versión francesa respecto a la obra original a consecuencia de haberse descuidado su carácter poético.

En cuanto a los estudios sobre traducciones al inglés, los tres que se incluyen versan sobre obras de Miró. David Bell presenta una visión crítica de las decisiones del traductor inglés durante su proceso de recreación de Figuras de la Pasión del Señor, concluyendo que el uso de un lenguaje arcaico en los diálogos (ausente en el texto español) tiene como resultado un claro distanciamiento respecto al estilo de la obra original. Adelina Gómez analiza en profundidad la traducción al inglés de las expresiones de emotividad de la novela El obispo leproso, prestando especial atención a los elementos que expresan la pequeñez, en concreto las 'palabras mínimas' y los sufijos diminutivos. Y Juan Jesús Zaro analiza dos traducciones al inglés de Nuestro Padre San Daniel, muy alejadas en el tiempo (1930 y 2011) y muy distintas entre sí, poniendo de manifiesto las estrategias de traducción de cada una para recuperar el léxico de índole cultural y los tropos, así como la dificultad de traducir la obra del escritor alicantino.

El último bloque reúne seis estudios sobre traducciones al checo, neerlandés, rumano y ruso de obras de ambos escritores españoles. Así, Jana Králová lleva a cabo un análisis meticuloso, en la versión checa de Los pueblos de Azorín, de la traducción del título, los adjetivos, la direccionalidad, los topónimos y la variación intralingüística, valorando el esfuerzo por introducir en la cultura checa un género, conceptos y formas poéticas que carecían de modelo autóctono en la época de su publicación. Vanda Obdržálková presenta un estudio riguroso del contexto histórico y cultural en que fue publicada la traducción checa de Figuras de la Pasión del Señor de Miró, de su traductor y de las características del método traductor. Hendrika Gevers ofrece un análisis detallado de la traducción al neerlandés de La ruta de Don Quijote de Azorín, mostrando con abundantes y elocuentes ejemplos que el traductor no ha hecho justicia al escritor español, tampoco a los receptores de llegada ni a su lengua. El trabajo contrastivo de Adelina Angheluță se centra en la traducción de la forma de cortesía española 'usted' en la versión al rumano de Las cerezas del cementerio de Miró, atribuyendo las consecuencias semánticas a la elección de un pronombre poco adecuado para mantener la distancia discursiva por la que se ha optado en el texto original. A su vez, Delia Prodan presenta un interesante estudio sobre la proyección que la obra azoriniana ha tenido en la cultura literaria de Rumanía. Finalmente, en el último trabajo de este bloque, y cerrando la segunda parte, Natalia Timoshenko analiza en profundidad el estilo, el vocabulario paisajístico y gastronómico, y ciertos problemas de orden cultural en la única traducción rusa de La ruta de Don Quijote.

La tercera parte recoge los testimonios y experiencias de los traductores vivos de Azorín y Miró. Así, el traductor norteamericano Walter Borenstein explica cómo descubrió a los dos escritores españoles y las principales dificultades estilísticas a que se enfrentó al realizar la traducción de algunas de sus obras. El traductor filipino Marlon James recuerda el momento en que aceptó, casi de forma casual, el encargo de traducir a Miró y su progresiva concienciación de la grandeza y el desafío que suponía la tarea encomendada. Por su parte, el traductor francés Christian Manso describe la ingente y ardua tarea que le supuso traducir a Azorín por la terminología, los elementos culturales y el ritmo de su prosa, a la vez que presenta la traducción como una aventura arriesgada del que uno nunca se siente completamente satisfecho. Y, por último, la traductora rumana Doina Lincu da cuenta de su descubrimiento personal de la obra de Miró y la fascinación que sintió desde el principio por el autor alicantino; asimismo, insiste en la constante preocupación del traductor que se pregunta por el acierto de su versión. Tal y como Fernando Navarro explica en el prólogo de la publicación, si bien no es habitual recabar la opinión del traductor en las contribuciones de crítica de traducciones, constituye un punto de vista sumamente importante e interesante por cuanto proporciona datos que permiten comprender mejor los entresijos de las obras traducidas y cómo los traductores los afrontan; de manera que esta última parte representa una de las aportaciones más novedosas del libro.

En conclusión, Azorín y Miró en traducción es una obra rigurosa, que conjuga unidad y diversidad, innovadora en varios aspectos y minuciosamente editada y revisada. El tema principal e hilo conductor -las obras de Azorín y Miró-, ya suficientemente interesante por sí mismo, es abordado desde una perspectiva poliédrica y multidisciplinar que lo enriquece. La variedad de enfoques metodológicos, así como las diversas especializaciones y combinaciones lingüísticas de los autores participantes, contribuyen a ofrecer una panorámica muy completa de la temática tratada y 
de algunas de las posibles líneas de investigación dentro de los estudios de crítica y traducción literaria. Asimismo, cabe destacar que el rigor científico de cada uno de los artículos se completa con el valor inestimable de la voz testimonial de los traductores vivos de Azorín y Miró. Así pues, nos atrevemos a augurar que, gracias a todas estas características, además de ser de gran ayuda para dar a conocer aún más la obra de los dos escritores españoles y a sus traductores, que los han acercado al público lector que no habla su idioma, este volumen será de gran interés no solo para los estudios de crítica y traducción literarias, sino también para los Estudios de Traducción en general. Sin duda, el coordinador de la publicación y el elenco de expertos que han participado en la misma merecen nuestro más sincero elogio por el trabajo realizado y confiamos en que su obra forme parte de la bibliografía recomendada en esta disciplina.

Montserrat Cunillera Domènech Universitat Pompeu Fabra, Barcelona, España

Lathey, Gillian (2016): Translating Children's Literature. Abingdon, New York: Routledge, $161 \mathrm{p}$.

L'ouvrage s'inscrit dans la collection des guides pédagogiques de Routledge «Translation Practices Explained», conçue pour accompagner étudiants, professeurs et traducteurs en complément de leurs cours, recherches ou travaux, et dirigée par Kelly Washbourne. Lathey y aborde différents aspects et stratégies de traduction en littérature enfantine, et propose à la fin de chaque chapitre, conformément à la construction des ouvrages de la série, des sujets de discussion et des exercices thématiques, ainsi que des lectures théoriques pour approfondir chaque sujet d'étude. Le guide s'articule autour d'une double trame constituée de sept chapitres traitant respectivement de la communication narrative avec le jeune lecteur (p. 15-36), de la rencontre avec «l'inconnu» (p. 37-54), des images (p. 55-70), des dialogues et des dialectes (p. 71-92), des sons (p. 93-112), des versions, de la retraduction et de la traduction-relais (113-126), et enfin de l'édition, de la mondialisation et du lectorat enfantin (p. 127-144). Les chapitres sont recoupés de grandes thématiques transversales: le discours, l'étranger, le rapport entre texte et images, la traduction en tant que projet, le rôle du traducteur et la réponse d'un jeune public rien moins qu'actif dans sa réception de l'œuvre traduite et parfois dans sa participation à la traduction.

Le discours est le premier grand fil conducteur de l'ouvrage, la communication narrative présentant plusieurs défis de taille: la nécessité de s'adresser à la fois aux lecteurs enfants et adultes dans la version cible si cette dualité discursive est présente dans l'original, la quête de la «voix narrative» juste, permettant de préserver la proximité avec la langue orale ou la richesse de l'ironie et de la connivence sous-entendues et la narration enfantine, défi que le traducteur surmontera en nourrissant sa propre culture en matière de littérature enfantine, et au contact d'enfants de l'âge du narrateur.

L’altération du discours par la censure au moment de la traduction ne concerne pas que les histoires émaillées de détails violents ou scatologiques comme les contes des frères Grimm, cette tentation persiste dès que les tabous et les sensibilités culturelles sont différents (p. 27).

Les dialogues, dialectes, l'argot et le parler enfantin sont abordés en détail dans le chapitre 4 . La traduction vers l'arabe ou l'hébreu soulève par exemple des problématiques comparables par l'existence d'une langue classique, de variantes de la langue parlée et d'une résistance historique à la vernacularisation, illustrée par la polémique autour de l'utilisation de l'«e-arabe» (Daoudi, 2011) dans le roman Banat Al Riyadh d'Al Sanea, et du choix des contraintes imposées aux traducteurs de Lindgren en hébreu (p. 73-74). Si l'argot mérite le respect de son contexte historique et de son origine sociale tout autant que le parler courant, comment rendre sa provenance géographique en traduction? Lathey évoque entre autres les défis de la traduction de l'argot berlinois des années 1920 d'«Émile et les détectives» de Kästner en comparant trois versions de la traduction vers l'anglais d'un court extrait du roman (p. 77): la version de Goldsmith (1931), caractérisée par un changement de registre langagier, celle de Massee (1930), la plus fidèle à l'original, et celle de Martin (2014), qui prend l'initiative de remplacer le parler enfantin du Berlin des années 1920 par celui des enfants américains d'aujourd'hui. La juste stratégie se décide au cas par cas, le sens de l'œuvre et la sensibilité du traducteur faisant loi. Si le «verlan» français de Kiffe Kiffe demain (Guène, 2004), traduit par Ardizzone, est remplacé dans sa version anglaise par l'argot du sud de Londres avec l'aide de «slang advisors» (p. 82), Bell choisit quant à elle de créer une «démotique non spécifique» pour traduire un ouvrage allemand du $19^{\mathrm{e}}$ siècle sur les pirates (p. 83).

La méthode du traduire à voix haute («translating "aloud" ") pour préserver l'impact du texte lu ou des dialogues est utilisée par de nombreux traducteurs chevronnés: Londsale-Cooper et Turner, les traducteurs britanniques de Tintin (Hergé), rapprochent la traduction des dialogues de la performance (p. 62, 63), une approche partagée par Crampton qui, fuyant l'usage des nouvelles 